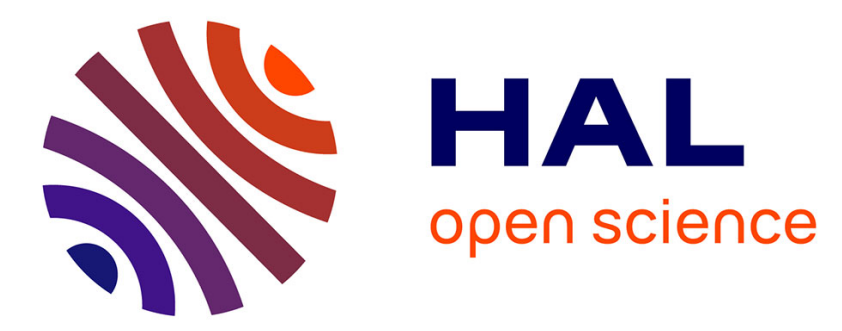

\title{
Les Marches de l'indépendance à Varsovie. Recompositions et transformations des droites extrêmes depuis les années 2000 en Pologne
}

Frédéric Zalewski

\section{- To cite this version:}

Frédéric Zalewski. Les Marches de l'indépendance à Varsovie. Recompositions et transformations des droites extrêmes depuis les années 2000 en Pologne. Cultures \& conflits, 2020, Le sens de l'action. Conflits de cadrages et interactions stratégiques, 117, pp.35-60. hal-02300945

\section{HAL Id: hal-02300945 \\ https://hal.parisnanterre.fr/hal-02300945}

Submitted on 30 Sep 2019

HAL is a multi-disciplinary open access archive for the deposit and dissemination of scientific research documents, whether they are published or not. The documents may come from teaching and research institutions in France or abroad, or from public or private research centers.
L'archive ouverte pluridisciplinaire HAL, est destinée au dépôt et à la diffusion de documents scientifiques de niveau recherche, publiés ou non, émanant des établissements d'enseignement et de recherche français ou étrangers, des laboratoires publics ou privés. 
Frédéric Zalewski

Institut des sciences sociales du politique, UMR CNRS 7220

Université Paris Nanterre

\section{Les Marches de l'indépendance à Varsovie Recompositions et transformations des droites extrêmes depuis les années 2000 en Pologne}

En Pologne, deux organisations d'extrême droite anticommuniste et néo-fasciste ${ }^{1}$, l'ONR (Obóz narodoworadykealny, Camp national-radical) et de la Jeunesse panpolonaise (Młodziė̇ $W$ szechpolska $)^{2}$, organisent chaque mois de novembre depuis 2010 une grande parade nationaliste dans les rues de Varsovie. Sous l'appellation volontairement apolitique de Marche de l'Indépendance (Marsz Niepodleglośsi), cette manifestation met à profit la date du 11 novembre, qui célèbre le rétablissement d'un État polonais en 1918. Les premières années, cette marche n'avait rassemblé que quelques centaines ou quelques milliers de participants, ne débordant ainsi guère des rangs des groupuscules d'extrême droite qui l'organisent. Après 2015, cependant, elle a progressivement pris de l'ampleur, au point de réunir près de 60000 personnes en 2017. Elle a alors attiré l'attention des acteurs politiques et des médias polonais, mais aussi des médias internationaux, lesquels ont promptement lié ce regain de fréquentation à l'arrivée au pouvoir du parti conservateur et souverainiste PiS à la fin de $2015^{3}$. Tout semble se passer, à leurs yeux, comme si le succès populaire de la marche, mais aussi son ambiance spectaculaire, entre geste fascisante et hooliganisme de supporters de foot, prenait son essor sur le terrain fertile de la rhétorique nationaliste et traditionaliste du PiS. En effet, dès son arrivée au pouvoir, ce parti a engagé la Pologne dans une dérive néo-autoritaire et anti-libérale ${ }^{4}$, s'appuyant sur des forces sociales conservatrices, en particulier sur l'Église ${ }^{5}$. Au-delà des conflits politiques qui ont matérialisé une évolution jugée anti-démocratique (sur l'indépendance des médias publics ou de la justice, dans les relations avec l'Union européenne), le PiS a ainsi été suspecté d'entretenir un climat favorable à la résurgence du fascisme en Pologne.

Tout en se situant dans une perspective d'analyse du basculement néo-autoritaire à droite après 2015, cet article entend dépasser cette lecture spontanée des marches d'extrême droite. Plutôt que d'y voir un symptôme de la présence du PiS au pouvoir, il propose d'en expliquer le succès par le travail de rénovation politique accompli par

\footnotetext{
${ }^{1}$ Un relatif consensus peut être observé, dans la littérature, pour distinguer, au sein de l'extrême droite, une frange néofasciste, qui, par rapport aux partis de droite radicale, se singularise par le maintien de références ou de pratiques directement héritées du fascisme. Cf. Piero Ignazi, Extreme Right Parties in Western Europe, Oxford University Press, 2003 ; Cas Mudde, Populist Radical Right Parties in Europe, Cambridge, Cambridge University Press, 2007. Le terme est entendu ici de façon descriptive et n'implique pas une approche typologique des groupes militants étudiés dans ce texte. Cf. Michel Dobry (dir.), Le mythe de l'allergie française au fascisme, Paris, Albin Michel, 2003.

${ }^{2}$ Ces deux groupes militants ont remis en usage des labels et des appellations solidement ancrés dans l'histoire du fascisme polonais. Le sigle ONR est mis en circulation par la droite nationale-catholique polonaise dès 1934, mais il fut repris par la suite par deux groupements d'inspiration fasciste, quoique toujours proches de la droite nationale-catholique, l'ONR ABC et l'ONR Falanga. La Jeunesse panpolonaise, créée en 1922, était affiliée à la droite nationale-catholique, dont elle était l'organisation estudiantine.
}

${ }^{3}$ Le PiS (Prawo i Sprawiedliwosc, Droit et Justice) est un parti politique conservateur, créé en 2001 par les frères Jarosław et Lech Kaczyński, anciens conseillers de Lech Wałęsa, leader de l’opposition démocratique polonaise des années 1980. Le PiS a brièvement exercé le pouvoir entre 2005 et 2007, mais Lech Kaczyński a été président de la République de 2005 à 2010 , laissant une empreinte durable sur la politique politique polonaise, en partie à cause des conditions traumatiques de son décès lors de la catastrophe aérienne de Smolensk. Vainqueur des élections législatives et présidentielle de 2015, le PiS reste dirigé par Jarosław Kaczyński. Sur le structuration du système de partis polonais après 2000, cf. Jérôme Heurtaux, La démocratie par le droit, Pologne 1989-2016, Paris, Presses de Science Po, 2017.

${ }^{4}$ Sur la situation polonaise post-2015, cf. entres autres : Georges Mink, «L'Europe centrale à l'épreuve de l'autoritarisme », Politique étrangère, n², 2016, p. 89-101 ; Frédéric Zalewski, "L’émergence d’une démocratie antilibérale en Pologne », Revue d'Études comparatives Est-Ouest, n4 (47), 2016, p. 57-86 ; Dorota Dakowska, «L'Europe centrale à l'heure du repli souverainiste », Études, n5, mai 2017, p. 19-30.

${ }^{5}$ Ewa Tartakowsky, Paul Zawadzki, «Politique et religion en Pologne », in: L'enjeu mondial, Paris, Presses de Sciences Po, 2017, p. 297-305. 
le nationalisme d'extrême droite polonais depuis la deuxième moitié des années 2000. Pour peu que l'on prenne an sérieux les tentatives des organisations d'extrême droite pour s'implanter dans la compétition électorale depuis les années 2010, ces défilés nationalistes du 11 novembre apparaissent comme une offre de respectabilité en direction des acteurs dominants de la droite conservatrice. De prime abord, cette thèse peut sembler difficile à soutenir puisque ces marches paraissent s'inscrire dans le registre de l'occupation de la rue, directement hérité des mouvements fascistes européens, et avoir dès lors vocation à contourner le jeu politique démocratique. Pourtant, les groupes nationalistes à l'origine de la Marche de l'Indépendance se sont avérés aptes à faire évoluer leurs modalités d'action et à revoir les conditions de leurs transactions avec les acteurs politiques dominants.

De faiblement institutionnalisé, éclaté entre plusieurs groupuscules de quelques dizaines ou au mieux quelques centaines de membres ${ }^{6}$, l'espace du nationalisme extrême droitier et/ou néo-fasciste ainsi s'est repolarisé vers la fin des années 2000 autour de l'ONR et de la Jeunesse panpolonaise, deux groupements militants nés après 1989. Les succès électoraux du parti d'extrême droite hongrois Jobbik, au cours des années 2000, semblent les avoir incité à se professionnaliser, ou plus exactement à produire une identité publique faite de sérieux et de respectabilité politique, impliquant une amorce de professionnalisation du groupe militant, ou tout du moins l'accumulation d'attributs de professionnalisation à son sommet ${ }^{7}$. Or, dans cette entreprise, les défilés de rue ont occupé une place centrale. Ils sont apparus disponibles pour investir la forme plus démocratique et légitime de la manifestation et équiper les situations de dispositifs propres à la politique professionnelle, comme des porteparole, des conférences de presse, comme une communication «moderne », à travers une forte présence sur les réseaux sociaux ou encore une couverture de l'événement par les médias dits «nationaux » proches de l'extrême droite.

Pourtant, ces marches nationalistes ne peuvent pas être réduites à un sous-produit des tentatives des organisations néo-fascistes de pénétrer dans le champ politique, dans une approche strictement stratégique. Elles ont en effet été au cœur d'un tournant observé ailleurs en Europe, caractérisé par l'émergence de l'identitarisme dans le discours politique comme dans l'offre idéologique des droites extrêmes. Dans le cas polonais, cette conversion à la question de l'identité, qui met à distance la matrice historique du fascisme ${ }^{8}$, a permis la construction et la diffusion de nouvelles narrations sur le présent et sur le passé, notamment en facilitant l'invention d'une nouvelle tradition «indépendantiste »" que les marches traduiraient en actes. Suffisamment floue dans ses contours pour être investie par des acteurs de la droite conservatrice conventionnelle et pour capter les enjeux de mémoire omniprésents dans la politique polonaise depuis les années 2000, cette nouvelle tradition est congruente avec les stratégies d'énonciation souverainistes et ethno-différentialistes de la nation, qui peuvent également traverser la droite conservatrice ${ }^{10}$. Si cet article entend souligner les limites d'une simple incrimination du « contexte », c'est ainsi parce que ces marches nationalistes participent au contraire activement d'une extension des domaines des droites, caractérisée par des appariement idéologiques et culturels inédits entre droite conservatrice et extrême droite ${ }^{11}$.

La démarche analytique de cet article est ainsi d'encastrer l'essor de la Marche de l'Indépendance dans des processus plus larges de transformation de l'extrême droite polonaise. En particulier, il se demande comment les mobilisations à l'origine de la Marche sont liées, empiriquement, à des conduites visant à travailler un rapport de force politique initial défavorable à l'extrême droite nationaliste, longtemps maintenue à la marge de l'espace politique par des scores électoraux insignifiants ${ }^{12}$. Dans une première partie, ce texte met ainsi en évidence

${ }^{6}$ David Ost, "The Radical Right in Poland: Rationality of the Irrational », in : Sabrina Ramet (dir.), The Radical Right in Central and Eastern Radical Europe since 1989, University Park, Pennsylvania State University Press, 1999. Voir aussi : Rafal Pankowski, The Populist Radical Right in Poland. The Patriots, Londres, New York, Routledge, 2010.

${ }^{7}$ Samuel Bouron, «Un militantisme à deux faces. Stratégie de communication et politique de formation des jeunesses identitaires », Agone, n² (54), 2014, p. 45-72.

${ }^{8}$ Enzo Traverso, Régis Meyran, Les nouveaux visages du fascisme, Paris, Textuel, 2017.

${ }^{9}$ Eric Hobsbawm, Terence Ranger, The Invention of Tradition, Cambridge, Cambridge University Press, 1983.

${ }^{10}$ Jean-François Bayart, L'illusion identitaire, Paris, Pluriel, 2018. Ce texte se détache ainsi des perspectives en termes de populisme, dominantes pour analyser les processus de droitisation nationaliste en Europe et, plus récemment, aux ÉtatsUnis. Sur l'imprécision de cette notion, cf. Damir Skenderovic, «Populisme», in : Olivier Christin (dir.), Dictionnaire des concepts nomades en sciences sociales, Paris, Métailié, 2010, p. 83-102.

11 Pour paraphraser Luc Boltanski et Arnaud Esquerre, Vers l'extrême. Extension des domaines de la droite, Paris, Editions Dehors, 2014. Ces modalités de reconfiguration idéologique ont par ailleurs été analysées par Stuart Hall, , Le populisme autoritaire. Puissance de la droite et impuissance de la gauche au temps du thatchérisme et du blairisme, Paris, ed. Amsterdam, 2008. Pour une analyse des transformations polonaises selon ce modèle, $c f$. Frédéric Zalewki, art. cit.

12 David Ost, art. cit. 
l'ampleur des transformations affectant l'ONR et la Jeunesse panpolonaise dès lors que ces deux formations souhaitent avoir accès au champ politique et se doter d'une image publique lissée, en apparence détachée de l'héritage néo-fasciste. Une deuxième partie s'arrête sur défilés nationalistes en eux-mêmes, pour en montrer la progressive transformation en «manifestation patriotique ». Enfin, une troisième partie interroge les transactions entre la droite conservatrice représentée par le PiS et la Marche de l'Indépendance, pour souligner l'ambivalence du PiS. Il s'agit plus spécialement de questionner les linéaments d'une stratégie de managed nationalism ${ }^{13}$ que celuici a tenté de mettre en place, notamment après la très forte visibilité médiatique de l'édition 201714.

\section{Les transformations de l'extrême droite nationaliste polonaise}

Les transformations de la droite extrême nationaliste et néo-fasciste concernent, en premier lieu, les formes mêmes du militantisme d'extrême droite, qui se tend à se professionnaliser et à intégrer des stratégies de visibilité médiatique, cruciale pour percer électoralement ${ }^{15}$. Conjointement, ces adaptations concernant les thématiques mises en avant, dans la mesure où leur idéologie anti-démocratique coûte fréquemment aux formations d'extrême droite leur participation effective à la compétition électorale. L'ONR et la Jeunesse panpolonaise ont ainsi répondu à ces contraintes par une inflexion progressive de leur discours vers le thème de l'identité, qui émerge en Europe comme réponse au multiculturalisme ${ }^{16}$. La valorisation de la nation, de sa transmission et de la mémoire de ceux qui se sont battus pour elle, à l'instar de la figure des soldats maudits ${ }^{17}$ - dont le culte déborde largement la sphère de l'extrême droite nationaliste - permet d'édulcorer la dimension idéologique du discours et de contester les accusations de fascisme.

La création de la Jeunesse panpolonaise remonte à1989. Elle a été en partie le fait d'un leader politique héritier d'une lignée de cadres de la droite nationaliste, Roman Giertych, qui en a été le premier président ${ }^{18}$. Ce dernier a également dirigé au cours des années 2000 la LPR ${ }^{19}$, un parti politique clérical et traditionaliste qui a participé à une coalition gouvernementale avec le PiS entre 2005 et 2007. À la création de ce parti, la Jeunesse panpolonaise en est devenue l'organisation de jeunesse et a constitué un vivier pour recruter des candidats aux élections. Ainsi, l'un des visages médiatiques de la Marche de l'Indépendance au cours des années 2010, Krzysztof Bosak (né en 1982) est devenu député LPR en 2005, après avoir été l'assistant parlementaire de Roman Giertych au Parlement européen puis de devenir président la Jeunesse panpolonaise en 2005-200620. Lors des législatives anticipées de

\footnotetext{
${ }^{13}$ Robert Horvath, «Russkii Obraz and the politics of "managed nationalism" ", Nationalities Papers, vol. 42, n³, p. 469-488.

${ }^{14}$ Ce texte présente une vocation exploratoire et se fonde sur des matériaux issus de la presse généraliste ou de la communication des groupes politiques étudiés, mais aussi sur des enquêtes antérieures sur la droite polonaise. $C f$. Frédéric Zalewski, art. cit. ; « Pologne : la contre-révolution des jeunes », Passages, n 95, 1999, p. 48-52 ; «Le phénomène populiste en Europe à l'épreuve des terrains : une comparaison entre le mouvement Autodéfense (Pologne) et le Parti Populaire Danois (Danemark) ", Revue Internationale de Politique comparée, vol. 12, 2005, p. 487-501 ; "Une droite anticommuniste "radicale" en Pologne? Lorsque les règles du jeu politique deviennent un trophée », in : Annie Collovald, Brigitte Gaïti (dir.), La radicalisation politique en questions, Paris, La Dispute, 2006.

${ }^{15}$ Ces processus de professionnalisation de l'extrême droite identitaire ont été étudiés en France et en Italie, voir : Samuel Bouron, «Un militantisme à deux faces. Stratégies de communications et politique de formation des jeunesses identitaires », Agone, n², 2014; p. 45-72 ; Samuel Bouron, Caterina Froio, «Entrer en politique par la bande médiatique ? Construction et circulation des cadrages médiatiques du Bloc identitaire et de Casapound Italia », Questions de communication, n²1, 2018, p. 209229 ; Pietro Castelli-Gattinara, Caterina Froio, «Quand les identitaires font la une. Stratégie de mobilisation et visibilité médiatique du bloc identitaire », Revue française de science politique, $\mathrm{n}^{\circ} 1,2018$, p. 103-119.

${ }^{16}$ Yannick Cahuzac, Stéphane François, "Les stratégies de communication de la mouvance identitaire », Questions de communication, $\mathrm{n}^{\circ} 23,2013$, p. 275-292.

${ }^{17}$ L'appellation de soldats maudits désigne depuis la fin des années 2000 les combattants de la résistance intérieure polonaise qui ont refusé de rendre les armes en 1945, lors de la prise de pouvoir des communistes. Fréquemment apparentés aux organisations politiques d'extrême droite, parfois auteurs d'exactions contre les populations civiles non polonaises, notammejils ont été sévèrement réprimés par le pouvoir communiste. Depuis 2010, une journée de commémoration leur est consacrée et leur évocation est devenue l'un des pivots de la nouvelle mémoire anticommuniste promue par la droite polonaise.

${ }_{18}$ Né en 1971, fils du militant nationaliste en émigration Maciej Giertych (né en 1936) et petit-fils de Jędrzej Giertych (19031992), ancien cadre politique de la droite nationale d'avant-guerre. Ancien ministre dans le gouvernement de coalition formé par le PiS entre 2005 et 2007, Roman Giertych est actuellement proche des libéraux de PO et, comme avocat, défend des personnalités ciblées par le law fair du PiS.
}

${ }^{19}$ Liga Polskich Rodziń, Ligue des Familles Polonaises.

${ }^{20}$ Michał Krzymowski, « Krzysztof Bosak, narodowiec-imprezowiec », Newsweek Polska, 18 novembre 2013. 
2007, la LPR a également présenté Robert Winnicki (né en 1985), qui a été président de la Jeunesse panpolonaise entre 2009 et 2013, période au cours de laquelle la Marche de l'Indépendance prend son essor. Toutefois, des tensions politiques fortes, liées à l'image d'extrémisme des Panpolonais, a conduit la LPR à créer une nouvelle organisation de jeunesse en décembre 2006. La Jeunesse panpolonaise s'est alors distanciée de la LPR et a recherché des alliances à l'extrême droite, notamment parce ses effectifs ont rapidement fondu après 2006 (en 2009, ses dirigeants revendiquaient à peine 200 adhérents) ${ }^{21}$.

L'ONR a émergé quant à elle dans les années 1990 au sein de la sous-culture skinhead, très politisée à l'extrême droite en Pologne, comme en Allemagne ou en Hongrie, bien que d'un poids largement inférieur à ces deux pays $^{22}$. Dès 1993, divers groupes locaux se sont affiliés à ce label, avant une progressive consolidation en 1999 et l'unification intervenue en 2005 autour du groupe local de Częstochowa. Depuis les années 2010, la direction de l'ONR s'efforce de se couper de ses membres affichant ouvertement leurs sympathies pro-nazies. Cette stratégie de relative modération la rend plus visible et lui permet notamment de contester à un autre groupement plus ancien, le NOP ${ }^{23}$, le leadership de l'extrême droite néo-fasciste. L'alliance nouée en 2010 avec la Jeunesse panpolonaise renvoie donc à un ensemble d'interdépendances concurrentielles entre groupes militants extrême droitiers, qui partagent à la fois des modes d'action mais aussi une origine commune dans la contre-culture skinhead, constituant un pôle spécifique de l'extrême droite polonaise.

Les liens entre la LPR et la Jeunesse panpolonaise attestent de la préexistence de ressources de professionnalisation, mais ce processus s'explique également par le recrutement social des groupes d'extrême droite. Comme dans les autres groupes identitaires européens, elle se manifeste par une élévation globale du niveau de compétences et d'expertise des militants situés au sommet de l'organisation ${ }^{24}$. Quelques exemples peuvent en attester : Krzysztof Bosak, président des Panpolonais en 2005-2006, est diplômé d'une école assurant des cours de communication et s'est familiarisé à l'univers des médias en étant candidat à la version polonaise du show télévisé Dancing with the stars; Robert Winnicki a fait des études de science politique à l'Université de Varsovie. Robert Bąkiewicz (né en 1976), qui a organisé les Marches de l'Indépendance au milieu des années 2010, met en avant des compétences de communication sur sa page personnelle Internet et a été candidat aux municipales de 2018 dans sa ville natale de Pruszków, près de Varsovie (voir encart 1) ${ }^{25}$. Comme dans le cas des Identitaires français, ces compétences de communication politique prennent place dans un processus plus large d'émergence de médias indépendants, affiliés directement aux groupes politiques d'extrême droite, dont les leaders sont les invités ou commentateurs réguliers et qui, en elles-mêmes, constituent des lieux où se roder à la prise de parole dans les médias (mentionnons par exemple les chaînes de télévision wRealu24 ou Telewizja Republika, ou encore la chaîne Youtube Media Narodowe lancée par l'association organisatrice de la Marche de l'Indépendance).

Encart 1 : une présentation de soi professionnalisée et dépolitisée

Candidat aux municipales de 2018 à Pruszków, Robert Bąkiewicz se présente aux électeurs par un bref texte qui atteste de la capacité à mettre en avant des éléments valorisés par le marketing politique, relatifs à la vie familiale et à la «proximité » avec l'électeur : «Je m'appelle Robert Bąkiewicz, j'ai 42 ans, je suis né et j'ai vécu toute ma vie à Pruszków. Je suis un époux comblé et le père de trois petites filles. La foi catholique romaine, dans laquelle ont été élevées des générations de Polonais, fonde ma morale. Je suis allé l'école primaire n¹0 et au lycée Zan de Pruszków, j’ai ensuite fait mes études à la PWSHiP. Actuellement je préside l'Association de la Marche de l'Indépendance et je suis directeur de la rédaction de la télévision Media Narodowe.

Je me caractérise par une riche expérience de conduite de projets et de gestion d'équipes, dont l'encadrement de la jeunesse. Je m'engage socialement dans les activités patriotiques, de bienfaisance et d'éducation principalement au niveau national. Mes atouts sont d'être travailleur, créatif et déterminé. ${ }^{26}$

\footnotetext{
${ }^{21}$ Wojciech Szacki, « Robert Winnicki, lider Wszechpolaków », Politykka, 20 novembre 2012.

22 Sur les cas allemand et hongrois : Gideon Botsch, «From skinhead-subculture to radical right movement: the development of a 'national opposition' in East Germany », Contemporary European History, vol. 21, n²4, 2012, p. 553-573 ; Laszlo Karsai, «The radical right in Hungary », in : Sabrina Ramet (ed.), op. cit., p. 133-146.

${ }^{23}$ Narodowe Odrodzenie Polski, Renouveau national de la Pologne.

${ }^{24}$ Samuel Bouron, Caterina Froio, art. cit.

${ }^{25}$ Page personnelle sur le site dédié aux élections dans cette ville : https://wybory2018.pruszkow.pl/kandydat-naprezydenta/robert-bakiewicz/ consulté le 5 septembre 2019.

${ }^{26}$ Ibid.
} 
En 2012, plusieurs militants et dirigeants de ces groupes extrémistes ont créé un parti politique, le RN27, destiné à être lancé dans la bataille des élections locales de 2014. Les animateurs du RN ont ensuite pu obtenir des positions éligibles sur certaines listes électorales aux législatives de 2015, comme Robert Winnicki ou Adam Andruszkiewicz (né en 1990), tous deux élus députés sur les listes du parti Kukiz'1528. Ces transformations amorcent l'émergence d'un pôle "national » ${ }^{29}$; elle se sont prolongées en 2019 avec la création d'une coalition électorale pour les élections européennes, la Confédération Liberté et Indépendance (Konfederacja Wolność $i$ Niepodległość). Cette nouvelle coalition s'est maintenue pour les élections de 2019, avec quelques modifications s'agissant des micro-partis qu'elle réunit, mais avec un rôle qui reste éminent pour le le RN. Elle a en l'occurrence été rejointe par un ancien candidat nationaliste à la présidentielle de 2015, Grzegorz Braun, tandis que l'exrappeur Liroy s'en détachait pour créer ses propres listes. Pour ces élections, la Confédération a également attesté de sa forte intégration politique en étant en mesure de présenter des listes dans toutes les circonscriptions.

La consolidation des groupes d'extrême droite élargit ainsi l'espace existant à la droite du PiS, en offrant des occasions supplémentaires de rattachement au champ politique et d'insertion dans la politique professionnalisée. Reprenons le cas déjà évoqué de Krzysztof Bosak : outre la LPR, celui-ci a été apparenté au parti de droite radicale Nouvelle Droite (Nowej Prawicy), fondé pour soutenir une figure centrale de la droite radicale polonaise, Janusz Korwin-Mikke ${ }^{30}$. Ce dernier, connu pour des positions ultra-conservatrices et libertariennes, ainsi que pour de nombreux «dérapages », a dirigé dans les années 1990 un parti représenté au Parlement, l'UPR ${ }^{31}$, a construit sa sa candidature à la présidentielle de 2015 en se rapprochant des groupements d'extrême droite, en répondant depuis des années à des invitations à des rassemblements «nationaux » organisés par l'ONR. En 2015, il a lancé un nouveau parti, KORWiN ${ }^{32}$, qui est devenu en 2019 l'une des principales composantes de la Cette trajectoire militante multi-positionnée a permis à Krzysztof Bosak de parvenir à une position éminente dans le $\mathrm{RN}$ lors de sa création, dont il reste vice-président, et de détenir un capital politique suffisant pour être parmi les quelques dirigeants qui, pour les législatives de 2019, construisent les listes et attribuent les positions jugées éligibles au sein de la Confédération Liberté et Indépendance.

Simultanément, l'inscription dans le registre idéologique de l'identitarisme s'est effectué en partie par une dénonciation de l'islamisation de l'Europe, mais aussi par une réécriture «nationale » ou « indépendantiste » de l'histoire de la Pologne. Cette réécriture n'est pas propre à l'extrême droite et relève en grande partie de la droite conservatrice, notamment du PiS, qui a développée une vision martyrologique de l'histoire récente, dans laquelle seules les années d'entre-deux-guerres ont été une expression authentique de la nation polonaise, à l'inverse de la période communiste. Ce basculement droitier prend entre autres la forme d'une réintégration progressive de la figure de Roman Dmowski (1864-1939), principal théoricien du nationalisme de la droite nationale, dans le panthéon des « grands hommes » de l'histoire polonaise de l'entre-deux-guerres. L'opposition canonique entre le «nationalisme fermé» de Dmowski et le «patriotisme ouvert» de Józef Piłsudki (1867-1935), homme d’État polonais des années 1910-1920 et figure qui a longtemps surclassé toutes les autres dans le panthéon du retour à l'indépendance en $1918^{33}$, est remplacée par une nouvelle synthèse qui agrège l'ensemble des principaux

\footnotetext{
${ }^{27}$ Ruch Narodowe, Mouvement national.

${ }^{28}$ Le parti Kukiz'15 a été créé par un ancien rockeur des années 1990, Paweł Kukiz, qui milite depuis le début des années 2010 pour une réforme électorale instaurant un scrutin uninominal de circonscription sur le modèle britannique pour mettre fin, selon les tenants de ce projet, au règne des partis. Proclamé pour cette raison «anti-système », son positionnement le met en situation de porosité avec la droite nationaliste, auprès de laquelle il a donc recruté des candidats aux élections, ne disposant d'aucune implantation territoriale développée.

${ }^{29}$ Le terme de narodowy («les nationaux »), qui est forgé sur le radical naród (nation, au sens de peuple), permet de se distinguer du lexique du nationalisme qui reprend le radical latin en vigueur dans d'autres langues, par exemple dans le terme même de nacjonalizm. L'appellation de Ruch Narodowy, adoptée pour le parti lancé après le succès des marches du 11 novembre, s'inscrit ainsi dans cette stratégie discursive. Sur la notion d'opposition nationale appliquée aux groupes néofascistes, $c f$. Gideon Botsch, art. cit.

${ }^{30}$ Sur Krzysztof Bosak, ex-jeune premier d'une émission de télé-réalité reconverti en politique, of le portait de Newsweek Polska: Michel Krzymowski, « Krzysztof Bosak, narodowiec-imprezowiec », Newsweek Polska, 18 novembre 2013.

31 Unia Polityki Realnej, Union de la politique réelle.

${ }^{32}$ Koalicja Odnowy Rzueczypospolitej Wolność $i$ Nadzieja, Coalition de Renouveau de la République Liberté et Espoir.

${ }^{33}$ Cette interprétation longtemps dominante est héritée de l'ouvrage d'Andrzej Micewski, Z geografii politycznej II

Rzecsypospolitej [De la géographie politique de la IIe République], Varsovie, ZNAK, 1964. Andrzej Micewski (1926-2004) était un
} 
dirigeants politiques de l'entre-deux-guerres. La Marche de l'Indépendance apparait alors comme l'espace où déployer avec une forte expressivité ces récits réécrits. Les supports visuels peuvent ainsi rapprocher les figures de Dmowski et Piłsudski, les associer à d'autres figures marquantes de la période, et procéder de la sorte à un syncrétisme qui gomme les divergences politiques et idéologiques souvent profondes sur la forme que devait prendre l'État polonais après l'indépendance. Ces transformations autorisent en particulier un classement de l'ONR dans la tradition «indépendantiste» ou "patriotique » et de prétendre en transformer la généalogie politique.

Enfin, ces transformations prennent également la forme d'un renouveau des liens internationaux. Au delà du seul Jobbik, qui a servi d'inspiration, les lien se sont renforcés au fil du temps avec le groupe néo-fasciste italien Force nouvelle (Forza Nuova). À titre d'exemple, la commémoration de la création de l'ONR de 2014 à Łódż s'est déroulée en la présence d'un délégué italien, Alessio Costantini, puis a donné lieu à la lecture d'une lettre du leader du parti nationaliste espagnol Démocratie national (Democracia Nacionah), Manuel Canduela ${ }^{34}$. La Marche du 11 novembre 2017 a amplifié cette tendance à l'européanisation des liens militants, avec la présence du leader de Forza Nuova lui-même, Roberto Fiore, de Manuel Canduela, encore, ainsi que du porte-parole du groupement suédois Jeunesse nordique (Nordisk Ungdom), Fredrik Hagberg ${ }^{35}$. Une invitation avait également été adressée à Richard Spencer, l'un des leaders les plus en vue du courant suprémaciste blanc américain, rattaché à l'alt-right ${ }^{36}$. La Marche du 11 novembre 2017 a été également rejointe par des représentants de partis d'Europe centrale et orientale, tel Milan Mazurek, du parti slovaque Notre Slovaquie (Naše Slovensko), dirigé par Marian Kotleba, ainsi que des militants hongrois ${ }^{37}$.

La densification des liens transnationaux à l'Est de l'Europe n'est pas sans conduire à des tensions idéologiques et à faire obstacle aux logiques d'harmonisation politique qui vont généralement de pair avec les processus de professionnalisation politique. L’un des segments militants représentés le 11 novembre 2017, les autonomes groupés autour de la publication Szturm (l'Assaut), a ainsi profité de l'occasion pour organiser les 10-11 novembre une conférence intitulée "L'Europe de l'avenir», largement axée sur des liens pan-slaves avec l'Ukraine mais aussi avec les pays baltes, et très critique sur la Russie de Poutine, suspectée de vouloir s'appuyer sur le déclin libéral du reste de l'Europe ${ }^{38}$. Mais à l'inverse, certains organisateurs de la marche se montrent, du fait de leur affiliation revendiquée au nationalisme polonais d'avant 1939, réservés sur les liens avec l'Ukraine, dont le nationalisme leur apparaît comme anti-polonais. Robert Bąkiewicz a par exemple confié ses réserves à la revue de droite conservatrice Fronda: «nous abordons certains éléments avec prudence - nous sommes critiques avec la politique historique des Ukrainiens qui glorifient pour l'instant l'OUN-UPA, Bandera ou Choukhevytch [des organisations et des leaders nationalistes à l'origine de massacres de Polonais pendant la Seconde Guerre mondiale] (...) A mon avis, nous devons nous rapprocher des Ukrainiens selon les possibilités mais nous montrer exigeants, ne pas nous laisser intimider par la menace d'une guerre avec la Russie (...). »39

historien proche des milieux catholiques indépendants et libéraux. Le succès de sa thèse repose en partie sur sa position indépendante de l'historiographie directement contrôlée par le régime, mais aussi de sa reprise par des historiens occidentaux eux-mêmes construits en figures d'autorités scientifiques en Pologne (cf. par exemple Norman Davies, Histoire de la Pologne, Paris, Fayard, 1986).

${ }^{34}$ Ibid.

${ }^{35}$ http://www.polsatnews.pl/wiadomosc/2016-11-10/narodowcy-z-wloch-hiszpanii-i-slowacji-pojda-w-marszuniepodleglosci/ consulté le 29 décembre 2017.

${ }^{36}$ Richard Spencer était cependant absent, suite à une interdiction de séjour prononcée par les autorités polonaises, officiellement pour des raisons d'ordre public.

37 « 11 listopada. Jeden z największych Marszów Niepodległości w historii. Policja zatrzymała 45 aktywistów Obywateli RP », Gazeta Wyborcza, 11 novembre 2017, http://wyborcza.pl/7,75398,22634227,11-listopada-jeden-z-najwiekszychmarszow-niepodleglosci-w.html, consulté le 29 décembre 2017 ; Stanley Bill, « Polish Nationalism on the March: The Context behind the Controversy ", art. cit.

${ }^{38}$ Compte-rendu en français sur le blog nationaliste ukrainien Reconquista : http://reconquistaeurope.tumblr.com/post/167820348256/lorganisation-nationaliste-polonaise-szturmowcy consulté le 29 décembre 2017.

${ }^{39}$ Luiza Dołęgowska, « Robert Bąkiewicz dla Frondy: Maszerowali patrioci, prowokatorzy darli mordy », en ligne sur http://www.fronda.pl/a/robert-bakiewicz-dla-frondy-maszerowali-patrioci-prowokatorzy-osmieszali-marsz-i-darligeby,102484.html consulté le 29 décembre 2017. 
Les manifestations du 11 novembre sont ainsi au centre d'un double mouvement, en étant un premier lieu une occasion de produire des dispositifs qui à attester d'une capacité à s'approprier les normes et les pratiques du champ politique, mais aussi, sur le plan idéologique, d'un recentrage de l'extrême droite sur des valeurs patriotiques, nationales, détachées en apparence du fascisme. Elles apparaissent ainsi comme la pierre de touche d'un basculement vers l'identitarisme et de nouvelles formes d'action politique en Europe, reléguant dans le passé le "nationalisme européen» élaboré en réponse à la guerre froide après 1945 dans l'extrême droite $\mathrm{d}^{\prime} E$ rope occidentale ${ }^{40}$ : à cet titre, elle intègre l'extrême droite polonaise dans un jeu identitaire largement globalisé.

\section{Les Marches de l’Indépendance, du défilé néo-fasciste à la manifestation « pacifique " ?}

L'histoire de la Marche de l'Indépendance peut être relatée sous l'angle des transformations des groupes qui en sont les organisateurs, mais aussi sous celui de la forme même qu'elle prend au fil du temps. Avant 2010, les groupements d'extrême droite polonais multipliaient les défilés et l'ONR, en particulier, mettait à profit diverses occasions de commémorations pour descendre dans la rue : date anniversaire du soulèvement de Varsovie en 1944, création de la première mouture de l'ONR dans les années 1930, par exemple. En 2010, le déroulement en Pologne de la marche LGBT de l'EuroPride avait offert l'occasion d'organiser une Marche de Grunwald (Marsz Grunwald ${ }^{(}(\imath)$, pour célébrer les 600 ans de cette bataille et pour illustrer la «défense des racines chrétiennes de l'Europe $»^{41}$. Les marches du 11 novembre étaient alors organisées par l'ONR, mais aussi par des groupes rivaux, comme le NOP (Narodowe Odrodzenie Polski, Renouveau national de la Pologne). Ces marches actualisaient un répertoire d'action et un imaginaire politique classiquement issus du fascisme : défilés en uniformes, drapeaux, chants nationalistes, etc. Encore en 2018, lors de la Marche de l'Indépendance qui s'est tenue à Wrocław, sur le modèle de celle de Varsovie, les manifestants ont scandé Wasže kamiennice, nasže ulice! (Vos immeubles, nos rues .).

La Marche de l'Indépendance s'inscrit dans cette tendance. Elle remonte aux années 1990 et s'est tenue pour la première fois à Varsovie en 1996, après quelques éditions les années précédentes dans des villes de province. Celles-ci étaient alors organisées par les skinheads d'un groupuscule nationaliste créé au début des années 1990 par un ancien membre de la frange conservatrice et nationaliste du parti communiste, Bolesław Tejkowski ${ }^{42}$. Sous sa forme actuelle, la Marche résulte des efforts conjoints de la Jeunesse panpolonaise et de l'ONR pour créer un cortège unique en lieu et place des défilés épars existant auparavant, qui ne réunissaient généralement qu'un très faible nombre de participants (voir tableau 1). La première marche conjointe s'est déroulée en 2010, puis l'année suivante ces deux groupes lui ont conféré une personnalité juridique nouvelle, en mettant en place une association chargée d'en coordonner les préparatifs et d'assurer une communication indépendante, l'Amicale de la Marche de l’Indépendance (Stowarzyszzenie Marsz. Niepodleglości).

Tableau $1:$ les effectifs rassemblés par la Marche de l'Indépendance (estimations de la police)

\begin{tabular}{|l|r|}
\hline 2009 & 300 \\
Organisée par la seule ONR & 2000 \\
\hline 2010 & $\begin{array}{l}\text { Co-organisée par l'ONR et la Jeunesse } \\
\text { panpolonaise }\end{array}$ \\
\hline
\end{tabular}

\footnotetext{
40 Jean-Yves Camus, Nicolas Lebourg, Les droites extrêmes en Europe, Paris, Seuil, 2015.

${ }_{41}$ Wojciech Karpieszuk, Piotr Machajski, «Europride z kontra », Gazeta Wyborcza, 16 juillet 2010. La bataille de Grunwald (1410) marque l'émergence de la Pologne-Lithuanie comme puissance européenne, grâce à une victoire décisive sur l'ordre des Chevaliers teutoniques. Elle est intégrée au récit national polonais depuis le 19e siècle.

42 Rafal Pankowski, op. cit., p. 86-87. Tejkowski fut dans les années 1950 proche des milieux marxistes dits révisionnistes et fut notamment l'assistant de Zygmunt Bauman à l'Université de Varsovie. Les skinheads membres de son organisation se distinguèrent dès les années 1990 par des marches ponctuées de violences, par exemple en 1992 à la frontière germanopolonaise, où furent brûlés des drapeaux allemands et israéliens et où des touristes allemands furent attaqués.
} 


\begin{tabular}{|l|r|}
\hline $\begin{array}{l}2011 \\
\text { Création de l'Association pour la Marche de } \\
\text { l'Indépendance }\end{array}$ & 7000 \\
\hline 2012 & 20000 \\
\hline 2013 & $50000^{*}$ \\
\hline 2014 & 25000 \\
\hline 2015 & 70000 \\
\hline 2016 & 75000 \\
\hline 2017 & 60000 \\
\hline
\end{tabular}

* Anticipations de la Ville de Varsovie d'après les déclarations légales des organisateurs. Sources: Gazeta Wyborcza, Newsweek Polska

La transformation tendancielle en manifestation s'est également produite à mesure que les relations avec les autorités (notamment celles de la ville de Varsovie, dirigées par les libéraux de la PO) se sont réglées. Les principaux désaccords concernaient le parcours de la Marche, après des incidents violents sur un axe nord-sud, en partie à cause de la nature des édifices et des monuments qui s'y trouvent (voir encart 1). Après les premières éditions, le parcours se fixe vers 2015 sur un axe ouest-est, à partir du rond-point Dmowski en plein centre-ville, jusqu'au stade national, situé sur la rive gauche de la Vistule, largement pavané aux couleurs nationales dans son parement extérieur. En 2017, la Marche a bénéficié pour la première fois du statut de rassemblement cyclique ${ }^{43}$, qui permet à des organisateurs de manifestations de préempter un parcours accordé par les autorités de police lors de rassemblements antérieurs et de bénéficier d'une protection spéciale contre les contre-manifestations.

Avant 2015, les défilés n'étaient pas eux-mêmes le point d'orgue de la journée de mobilisation, puisque les organisateurs prévoyaient également des rassemblements statiques pour permettre des prises de parole. En 2013, ces deux moments distincts avaient ajoutés à la confusion, puisque la ville de Varsovie avait interdit la manifestation suite à des échauffourées mais avait maintenu le meeting politique, ce qui avait conduit à un prolongement des troubles tard dans la soirée. À partir de 2015, et plus encore lors de l'édition 2017, c'est la manifestation qui constitue l'essentiel des mobilisations. Elle n'est suivie que de brèves prises de parole devant le stade national. La manifestation se veut ouverte à tous les "patriotes» et se décompose ainsi en différents publics ou différents segments militants. L'ONR et la Jeunesse panpolonaise ne sont pas les seuls représentés. D'autres groupements forment leurs propres cortèges, auxquels ils impriment leur marque. Par son ampleur, la manifestation devient également le réceptacle du syncrétisme croissant, qui n’est pas propre à la Pologne, entre supporters de football et groupes militants d'extrême droite ${ }^{44}$. La mise en scène du "patriotisme» des manifestants se déploie ainsi à travers le registre du supporterisme des stades : fumigènes, style vestimentaire ${ }^{45}$ (écharpes de clubs, mode identitaire, notamment), recul de la discipline stricte des défilés para-militaires. Des pratiques plus individuelles se greffent en outre sur le cortège, comme celle des déguisements avec des costumes

\footnotetext{
${ }^{43}$ Ce statut a été créé en 2016 en grande partie pour empêcher toute entrave aux commémorations mensuelles de Smolensk.

Cf Michal Szflarski, «11 listopada. Marsz Niepodległości zarejestrowany na cztery lata », Gazeta Wyborcza, 7 novembre 2017, http://warszawa.wyborcza.pl/warszawa/7,54420,22616604,11-listopada-marsz-niepodleglosci-zarejestrowany-nacztery.html consulté le 3 janvier 2018.

44 Sur les transformations du supporterisme et les stratégies des organisations d'extrême droite pour investir les espaces de sociabilité populaire liés au football en Europe, of. Richard Giulianotti, John Williams (dir.), Game without Frontiers. Football, Identity and Modernity, Aldershot, Arena, 1994 ; Lynda Dematteo, "Le stade, terrain de jeu de l'extrême droite italienne : soupape de sécurité ou fabrique du consensus ? ", L’Homme et la Société, 2015, n¹, p. 147-174 ; Mickaël Correia, Une histoire populaire du foorball, Paris, La Découverte, 2018.

${ }^{45}$ Sur l'émergence d'une mode identitaire parmi les jeunes gens d'extrême droite, voir l'étude du cas allemand par Cynthia Miller-Idriss, The Extreme Gone Mainstream, Commercialization and Far Right Youth Culture in Germany, Princeton, Princeton University Press, 2018.
} 
d'époque, suivant une pratique qui rappelle les reconstitutions historiques d'amateurs (prédominent les uniformes de la Première Guerre mondiale, polonais mais aussi français en référence à la mission Weygand ${ }^{46}$ ).

Dans le même mouvement, les organisateurs choisissent chaque année une devise pour donner une coloration spécifique à la Marche. Celles-ci puisent dans un imaginaire ancré dans le nationalisme polonais, au détriment des mots d'ordres ouvertement néo-fascistes : «Nous voulons Dieu» (Chcemy Boga) en 2017 ou «La Pologne aux Polonais, les Polonais pour la Pologne» (Polska dla Polakón, Polacy dla Polski) en 2015, par exemple. Ces devises sont l'un des supports permettant une communication adaptée à l'ère médiatique et numérique : site Internet spécifique, communication léchée reprenant les conventions du marketing politique, clips promotionnels à la façon d'Hollywood, prises de parole de parrains politiques, tel le sénateur PiS Jan Żaryn, sur Youtube, etc ${ }^{47}$. Ces mots d'ordre témoignent, encore une fois, de l'émergence d'une grammaire identitaire qui n'est pas propre à la Pologne. En 2013, le slogan choisi était « Une nouvelle génération est en marche » (Idziie nowe pokolenie), qui n’est pas sans évoquer l'un des aspects du lissage de son image par l'extrême droite identitaire, qui entend suggérer un renouveau patriotique dont la jeunesse serait porteuse. En 2016, la devise de la Marche avait été «la Pologne, citadelle de l'Europe» (Polska bastionem Europy), en référence à la bataille de Vienne en 1683 contre l'Empire Ottoman. Cette devise avait permis de mettre en scène les célèbres hussards ailés du roi Jan Sobieski, mais dont l'image d'Épinal est également reprise par les Identitaires autrichiens.

Davantage que d'entériner la transformations des anciens défilés néo-fascistes en simple manifestation, ce travail de communication et d'ouverture à de nouveaux publics manifestants conduit à une mise en tension des modèles d'occupation de la rue hérités de l'ancienne matrice d'action néo-fasciste. À titre d'exemple, le groupe Szturm ('Assaut), qui rassemble des autonomes d'extrême droite, occupe la rue en s'inspirant des modalités d'occupation de la rue des black blocks. En 2017, c'est de la part de ces groupements non affiliés que seraient venus les slogans les plus virulents et racistes tenus durant la Marche, dont on peut citer quelques exemples : «Sang pur et

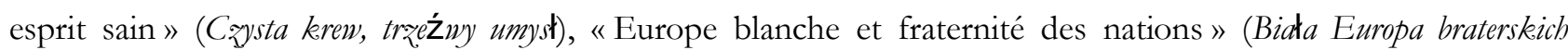
narodów), «L'Europe sera blanche ou ne sera pas » (Europa będzie biała albo bęludna), « Le nationalisme est notre voie » (Naszą drogą nacjonalizm), «Mort aux ennemis de la patrie » (Śmieŕ́ wrogom ojcayżny), Sig Heil, Ku Klux Klan, etc $^{48}$. Largement relevés par la presse, ils avaient contraint les organisateurs à se justifier publiquement du racisme sous-jacent à leur manifestation.

\section{Encart 2}

Les violences commises en marge de la Marche de l'Indépendance de 2013

La Marche de l'Indépendance 2013 offre un exemple des violences commises en marge du cortège, attestant de la difficulté des organisateurs à encadrer les conduites individuelles et à apparaitre comme un interlocuteur «fiable » auprès des autorités (en 2013, les libéraux de la Plateforme civique PO contrôlent à la fois le gouvernement et la mairie de Varsovie). La confusion qui s'installe au fil de la journée rend le décompte précis des manifestants incertain et la presse ne fait état que du nombre de participants déposé auprès des services de la Ville de Varsovie. La manifestation débute vers $15 \mathrm{~h}$ par des discours nationalistes ponctués de slogans tels que «fierté, fierté, fierté nationale » (Duma, duma, duma narodowa). Vers 16h00, les premières échauffourées se produisent avec la police (charges de la police, voitures incendiées) puis vers 16h30, les manifestants incendient une installation d'art contemporain intitulée Tęcza, figurant un arc-en-ciel, située sur le parcours, aux cris de « un garçon, un fille, une famille normale » (Chłopak, dziewczyna, normalna rodzina) et «Interdit de pédaler» (Zakaz pedalowania). De petits groupes de manifestants empruntent les rues perpendiculaires pour se diriger vers un squat anarchiste, attaquée à coups des projectiles ; les occupants se défendent depuis le toit par des jets de bouteilles et de pavés. À partir de 17h00, une série d'attaques se produisent contre l'ambassade de Russie, qui

\footnotetext{
${ }^{46} \mathrm{Du}$ nom de la mission militaire inter-alliée en Pologne après la Première Guerre mondiale, pour porter assistance au jeune État polonais dans sa guerre contre la Russie bolchevique.

${ }^{47}$ L'émergence de ce qui est appelé dans le contexte français les «fachosphères » reflète la possibilité pour les groupes politiques d'extrême droite de compenser leur faiblesse numérique par un activisme accru sur les réseaux sociaux. $C f$. Samuel Bouron, art. cit.; Robert Horvath, "Russkii Obraz and the politics of "managed nationalism" ", Nationalities Papers, vol. 42, n³, p. 469-488.

48 Jack Harlukowicz, http://wyborcza.pl/7,75398,22637091,na-marsz-niepodleglosci-przyszli-z-haslem-o-bialej-europie.html consulté le 3 janvier 2018. Le même article souligne que les slogans Ku Klux Klan ou Sig Heil n'ont été attestés que par un seul journaliste présent sur place.
} 
se situe sur la suite du parcours, obligeant la police à se redéployer. À 17h30, les autorités locales décident de dissoudre la manifestation ; la confusion s'installe : les attaques contre l'ambassade de Russie se prolongent jusque dans la soirée, une partie des manifestants se dirige vers un nouveau lieu de rassemblement, ayant fait l'objet d'une déclaration séparée par les organisateurs. Ces derniers prétendent par ailleurs ne pas avoir été informés de l'interdiction de la manifestation. Vers 19h40, les manifestants se dispersent - trente personnes ont été arrêtées, sept policiers blessés. Dans la soirée, le ministère des Affaires étrangères réagit aux violences commises devant l'ambassade de Russie et le premier ministre, Donald Tusk, condamne les exactions. L'intensité des violences et les réactions officielles amorcent les reprises médiatiques et politiques qui se multiplieront les années suivantes. À la télévision, Krzysztof Bosak, de la Jeunesse panpolonaise, déclare à propos de l'interdiction prononcée par les autorités locales : «la Marche est pacifique et la Ville de Varsovie a commis une erreur ${ }^{49}$.

D’autres tensions résultent du caractère hasardeux de la professionnalisation de la communication accompagnant la Marche. En 2017, le porte-parole de la Jeunesse panpolonaise, Mateusz Pławski (né en 1993), interrogé par l'hebdomadaire de droite Do Rzeczy sur la fréquence de slogans tels Sieg heil au cours de la manifestation, déclarait par exemple : «Nous ne sommes pas des racistes, mais des séparatistes de races ${ }^{50}$. Le lendemain, la Jeunesse panpolonaise devait ainsi se désolidariser de son porte-parole et le remplacer.

Ces tensions se répercutent sur la marche elle-même, qui reste émaillée d'incidents violents d'années en années. Les organisateurs dénoncent alors les «provocations » de la police ou des « gauchistes » pour les expliquer, dans la mesure où les premières éditions de la marche avaient d'emblée suscité des contre-manifestions d'antifas. Les différentes éditions ont été marquées par des violences récurrentes envers la police, par des dégradations de mobilier urbain, par des incidents avec des contre-manifestants. En 2011 et 2012, les manifestants s'étaient attaqués à des véhicules de police ou de télévision, dont certains avaient été incendiés. Après 2015, les actes de violence ont vu leur forme et leur signification s'infléchir, car ils sont entrés en résonance avec le nouveau rapport de force politique créé par l'arrivée au pouvoir du PiS. La Marche de 2017 a ainsi été marquée par le saccage du lieu de recueillement dédié à Piotr Szczęsny ${ }^{51}$, mais aussi par des violences physiques contre des militantes des groupes militants engagés dans la défense de l'État de droit en Pologne (cf. encart 3). En 2018, les Citoyens de la République ${ }^{52}$, ont été tenus à bonne distance du cortège par un cordon de police, mais ont été agressés verbalement et par des pétards ou fumigènes

Le succès de la Marche (en termes de fréquentation, de reprises médiatiques, de visibilité internationale) fait ainsi sortir l'ONR et la Jeunesse panpolonaise des marges de l'espace politique dans lesquelles se trouvaient les organisations néo-fascistes et/ou extrême droitière jusqu'à la fin des années 2000. Ce succès, cependant, s'accompagne des tensions propres à l'entrée dans un jeu politique élargi, professionnalisé et soumis à des injonctions plus fortes de respect de la norme démocratique. Ces tensions se maintiennent alors même que la question de l'identité se diffuse pourtant largement dans la droite polonaise, notamment au sein du PiS.

\section{Les transactions politiques entre la droite conservatrice et la droite « nationale »}

\footnotetext{
${ }^{49}$ « Marsz Niepodleglosci 2013 : Marsz rowiazany, Tusk przeprasza Warszawiakow », Polskatimes.pl,, 11 novembre 2013 , https://polskatimes.pl/marsz-niepodleglosci-2013-marsz-rozwiazany-tusk-przeprasza-warszawiakow-relacja-video-

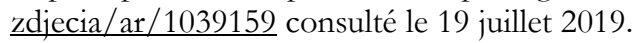

50 « Nie jesteśmy rasistami, ale rasowymi separatystami», Do Rzeczyy, 12 octobre 2017. Cette citation est en réalité le titre choisi par la rédaction de l'hebdomadaire en condensant les propos tenus pendant l'entretien. Les déclarations de Mateusz Pławski sont les suivantes : "nous pensons que la variable des races, la variable ethnique est très importantes, mais nous ne sommes pas racistes (...). Dans le slogan "Europe blanche”, il n'y a à notre avis rien de mal. Nous sommes des séparatistes de races, nous ne pensons pas qu'il y a des races supérieures aux autres mais elles sont toutes différentes et appartiennent à des continents différents. L'ethnicité ne doit pas être mélangée. »

${ }^{51}$ Né en 1963, Piotr Szczęsny était un simple particulier sans engagement politique qui s'est immolé par le feu à Varsovie en 2017 en signe de protestation contre la dérive autoritaire du PiS. Une plaque commémorative, fréquemment accompagnée de petits ex voto populaires, signale l'endroit où il s'est donné la mort.

52 Citoyens de la République (Obywatele RP) : groupe civique formé en 2016 pour s'oppose raux commémorations mensuelles de la catastrophe de Smolensk.
} 
L'émergence d'un pôle extrême droitier qui se revendique «national» au sein du champ politique conduit à un basculement des relations de concurrence dans lesquelles étaient auparavant pris l'ONR et la Jeunesse panpolonaise : c'est désormais moins avec les groupes néo-fascistes qu'avec la droite conservatrice que ces relations se nouent, de même qu'avec un espace semi-professionnalisé entre droite conservatrice et extrêmedroite, bien incarné par le cas déjà évoqué de Korwin-Mikke. Les conditions de cette concurrence sont néanmoins totalement différentes, puisque l'enjeu est bien d'émerger comme pôle autonome face au PiS, qui entend limiter la concurrence sur sa droite mais qui contribue activement à l'émergence du discours identitaire très distinctif pour cette nouvelle droite « nationale».

Avant 2015, les collusions entre le PiS et les mobilisations donnant lieu à la Marche de l'Indépendance prenait la forme assez élémentaire de ralliements individuels, de la part d'élus locaux ou nationaux du PiS ou d'autres acteurs de la droite polonaise. Au fil des ans, les soutiens se sont accumulés, parmi lesquels Paweł Kukiz, déjà mentionné pour avoir accueilli des membres du RN sur ses listes lors des élections législatives de 2015. L’un des soutiens les plus significatifs a été représenté par le sénateur PiS Jan Żaryn. Dès avant 2015, celui-ci signifiait publiquement son soutien aux défilés successifs, en enregistrant notamment de courtes prises de parole diffusées ensuite sur Youtube par les organisateurs de la Marche. Or, Jan Żaryn, par ailleurs historien de profession, est l'un des « entrepreneur de mémoire » les plus actifs du PiS et apparaît comme est l'un des propagateurs d'un récit national positif, condamnant toute forme d'histoire critique ${ }^{53}$. Son engagement parait ainsi illustrer les collusions entre le discours du PiS, refusant la "pédagogie de la honte», expression qui désigne en Pologne la « repentance », conduisant entre autres à une réhabilitation de la droite nationale antisémite d'avant-guerre, et la Marche de l'Indépendance, qui insiste sur les héritages politiques nationalistes polonais, au détriment de références ouvertement néo-fascistes.

L'arrivée du PiS au pouvoir en 2015 bouleverse cependant la structure des transactions entre ce parti et la marche. Le PiS s'est en effet trouvé contraint de prendre position et d'engager l'ensemble de sa politique gouvernementale dans un soutien ou à l'inverse dans une condamnation de la Marche de l'Indépendance. Le PiS a ainsi adopté une stratégie de jeu sur les limites, consistant à concilier différents objectifs : capter certaines rétributions de l'expression ouverte de nationalisme qu'est la Marche, en soulignant son "patriotisme », maintenir en apparence les principes établis par les gouvernements antérieurs de lutte contre l'extrémisme tout en faisant porter une partie de la critique vers l'opposition pour en dénoncer la véhémence et l'hostilité aux expressions de "patriotisme ». En dépit de l'autonomie des sphères militantes constitutives de l'ONR et de la Jeunesse panpolonaise par rapport au PiS, la configuration de jeu n'est pas sans gagner en intelligibilité à la lumière de la notion de managed nationalism, destinée à mettre en évidence les stratégies de contrôle des mobilisations nationalistes extrémistes par des pouvoirs néo-autoritaires usant eux-mêmes d'une rhétorique nationaliste ${ }^{54}$.

Après 2015, le PiS est ainsi resté, aux yeux de l'opposition libérale, inerte face à l'ONR, voire a semblé faire preuve d'une relative bienveillance à son égard, alors que son caractère extrémiste paraissait avéré. L'interdiction de l'ONR s'est imposé comme l'un des enjeux des les transactions entre le PiS et l'opposition, à la suite de la multiplication d'incidents impliquant l'ONR. En effet, l'intensification du militantisme civique après 2015 conduit à une forte présence dans le rue de groupes de défense de la démocratie, dont le Comité de défense de la démocratie (KOD) ${ }^{55}$. En 2016, par exemple, les commémorations de la mort de la résistante Inka ${ }^{56}$ avaient donné lieu, à Gdansk, à une très vive altercation entre militants de l'ONR et militants du KOD, au cours de

\footnotetext{
${ }^{53}$ Jan Żaryn a effectué une partie de sa carrière professionnelle d'historien auprès de l'Institut de la mémoire nationale (IPN), où il a par exemple été directeur du bureau à l'éducation publique entre 2006 et 2009. Il a également été actif dans la création de la journée annuelle de commémoration des soldats maudits. Cf. Valentin Behr, «Genèse et usages d'une politique de l'histoire. La "politique historique” en Pologne », Revue d'études comparatives Est-Ouest, n³, 2015, p. 21-48.

${ }^{54}$ Robert Horvath, art. cit.

${ }^{55}$ Komitet Obrony Demokracji, Comité de défense de la démocratie. Fondé en 2015, le KOD a initié la vague de militantisme civique qui s'oppose au pouvoir de Droite et Justice. Au cours de l'année 2016 organisé d'imposantes manifestations en Pologne à la suite des premières tentatives de réformes du Tribunal constitutionnel, appréhendées par les oppositions comme des atteintes à l'État de droit.

${ }^{56}$ Danuta Siedzikówna (1928-1946) était une infirmière de la résistance polonaise (pseud. Inka), qui après la fin des combats contre l'Allemagne a rejoint les rangs des unités poursuivant la lutte armée contre les nouvelles autorités communistes. Arrêtée en 1946, elle fut exécutée la même année. Figure longtemps oubliée, son culte nouveau accompagne l'hérö̈sation des soldats maudits.
} 
laquelle la police avait dû s'interposer. Quatre députés libéraux de la PO avaient alors saisi le parquet pour faire interdire l'ONR. Mais la ligne de conduite du PiS avait maintenu une certaine symétrie entre ces deux protagonistes : le ministre de l’Intérieur Mariusz Błaszczak et la Première ministre Beata Szydło avaient ainsi qualifié les agissements du KOD de provocation délibérée ${ }^{57}$.

Lors de la Marche de l'indépendance de 2017, en dépit de l'affluence à la marche et de la visibilité accrue de ses dérives xénophobes et suprémacistes, le PiS a dans un premier temps tenu cette ligne. Au cours d'une conférence de presse, le ministre de l'Intérieur Mariusz Błaszczak a déclaré que la Marche devait être décrite avec impartialité : «la fête de l'indépendance s'est déroulée dans un climat satisfaisant, en toute sécurité, on pouvait voir du rouge et du blanc dans toutes les rues de Varsovie. C'était une vision réjouissante. $\aleph^{58}$. Ce thème a été développé dans les jours suivants, toujours pour récuser toute perspective d'interdiction de l'ONR. Questionné à ce sujet lors d'une émission télévisée, le Maréchal de la Diète Ryszard Terlecki a par exemple déclaré que les demandes d'interdiction de l'ONR, de la Jeunesse panpolonaise et de la Marche elle-même étaient " absurdes » et a ajouté : « je suis contre ces interdictions et pour que la Marche ait lieu (...) c'est une belle fête, la plus grande fête patriotique d'Europe $\aleph^{59}$. Jaroslaw Kaczyński a lui-même contribué à ce positionnement public, en déclarant que les débordements antisémites et racistes de la manifestation étaient « la marge de la marge » ${ }^{60}$.

\title{
Encart 3
}

«Plus grande manifestation fasciste d'Europe » : la Marche de l'Indépendance de 2017 vue depuis les contremanifestations civiques

\begin{abstract}
Alors que les organisateurs de la Marche mettent en avant son «patriotisme», les imputations de «fascisme» sont à l'origine de contre-manifestations. En 2017, à l'instar des premières années de la Marche, des groupes antifascistes organisent quelques rassemblements, mais d'autres contre-manifestations sont le fait des groupements militants nés des protestations civiques contre le PiS. Ainsi, dès $15 \mathrm{~h} 00$, un piquet se forme non loin du passage du cortège, organisé par les Citoyens de la République. Formé d'une trentaine de personnes, ce groupe reste à l'écart de la manifestation et déploie des banderoles dont les mots d'ordre dénoncent la dimension fascisante de la manifestation. Il est rapidement dispersé par la police, avant que ses participants ne soient mis à l'écart et surveillés par les policiers en charge de protéger le cortège de la Marche puis, pour certains, déplacés vers des locaux de la police. L'autre groupe de contre-manifestants a été formé par quatorze militantes de Protestation des Femmes (Protest Kobiet, association civique née en 2016 à la suite des tentatives de restriction du droit à l'avortement) et de militantes de Citoyens de la République. À la différence du groupe précédent, ces militantes vont au contact des manifestants et, portant une banderole «les femmes contre le fascisme», organisent un sit-in sur le pont Poniatowski, en un point plus étroit du parcours. Leur protestation a fait l'objet d'une préparation minutieuse, certaines militantes portent ainsi des perruques ou ôtant leurs lunettes pour ne pas être reconnues par la police. Pendant quelques minutes, elles déploient leur banderole de près de sept mètres de large sans réaction de la foule. Puis pendant une vingtaine de minutes, elles subissent les agressions verbales et physiques des manifestants (coups, crachats, insultes sexistes, arrachage de leur banderole, drapeaux polonais portés au visage en signe de défi), malgré la protection du service d'ordre de la Marche. Ces contremanifestations se sont distinguées des rassemblements statiques organisés par les groupes antifascistes dans la mesure où elles ont été en partie dirigées contre le PiS, à travers une mise en question du statut de rassemblement cyclique accordé à la Marche. La décision des militantes féministes de se rendre sur le tracé lui-même provenait d'un refus explicite de voir tenues à distance. Les contre-manifestants incriminent également les agissements de la police : lors de leur intervention contre le rassemblement statique des Citoyens de la République, les policiers établissent ainsi des procès-verbaux pour diffusion de «contenus fascistes » (une pancarte montrait une photo du
\end{abstract}

\footnotetext{
${ }^{57}$ https://www.tvn24.pl/wiadomosci-z-kraju,3/onr-i-kod-skladaja-zawiadomienia-do-prokuratury-ws-pogrzebuinki,672578.html consulté le 2 février 2018.

${ }^{58}$ https://oko.press/swieto-niepodleglosci-cieniu-rasizmu-pis-wychodzi-posiedzenia-europarlamentu-kronikaskorzynskiego-11-17-listopada-2017/ consulté le 2 février 2018.

${ }^{59}$ http://wiadomosci.radiozet.pl/Polska/Terlecki-Pomysl-delegalizacji-ONR-MW-i-Marszu-Niepodleglosci-to-nonsens consulté le 2 février 2018.

${ }^{60}$ https://oko.press/swieto-niepodleglosci-cieniu-rasizmu-pis-wychodzi-posiedzenia-europarlamentu-kronikaskorzynskiego-11-17-listopada-2017/
} 
procès de Nuremberg et une photo d'un rassemblement de l'ONR dont les participants faisaient le salut fasciste $)^{61}$.

En 2017, le PiS n'a revu sa position que sous l'effet de l'ampleur des critiques et de la visibilité internationale de la marche. Dès le 13 novembre 2017, le président de la République, Andrzej Duda, s’est exprimé publiquement pour dire que l'événement avait été dévoyé par des slogans condamnables, relevant du nationalisme davantage que du patriotisme : «le nationalisme est une forme d'expression négative, qui implique que notre pays n’est que pour nous. Ce n'est pas juste. Notre pays est pour tous ceux qui veulent y vivre honnêtement et qui veulent le construire. (...) Dans notre pays, il n'y a pas de place ni de consentement pour la xénophobie. Dans notre pays, il n’y a pas de place pour le nationaliste maladif » ${ }^{62}$. Les déclarations déjà évoquées de Jarosław Kaczyński étaient en fait assez proches, puisque celui-ci a estimé que « la tradition polonaise dont nous nous réclamons n'a rien à voir avec l'antisémitisme, nous sommes très éloigné de cela, très éloigné du racisme et de la xénophobie ${ }^{63}$. Au cours de cette période, Andrzej Duda présidait par ailleurs le comité d'organisation des commémorations officielles du centenaire de l'indépendance, prévue pour 2018. Or, quelques jours après les violences de la manifestation du 11 novembre 2017, Andrzej Duda a décidé d'écarter toute participation des animateurs officiels de la Marche au comité d'organisation du centenaire.

En 2017, Robert Bąkiewicz, le président de l'Association de la Marche de l'Indépendance, revenait sur les invitations adressées depuis 2015 à Andrzej Duda, président PiS : «depuis 2010, la Marche de l'Indépendance nous a permis de jouer un rôle important auprès de la nation polonaise, un rôle très important même, et nous ne voulons pas être relégués à un rôle secondaire, mais plutôt être considérés comme des partenaires. Bien sûr, ce serait un honneur pour nous que le président soit présent à la Marche, cela donnerait à celle-ci davantage d'importance. Mais nous ne voulons pas être des faire-valoir pour lui, plutôt qu'il soit l'invité le plus important ${ }^{64}$. En 2015 comme en 2016, Andrzej Duda avait fait parvenir un message de soutien aux manifestants, donnant lieu chacun à une lecture publique ${ }^{65}$. Ces déclarations ne peuvent mieux illustrer les usages de la Marche comme offre de respectabilité en direction de la droite conservatrice, mais ces efforts ont été réduits à néant par la scandalisation de la Marche en 2017.

Davantage qu'un soutien explicite et inconditionnel, ou qu'une permissivité aveugle, le PiS semble ainsi tenter d'intégrer les défilés nationalistes dans ses différentes narrations, sur la mémoire, sur la nécessaire renaissance de la nation ou encore sur l'hostilité de l'opposition libérale au fait national polonais. Il s'agit ainsi d'ajustements, qui reflètent également des tensions internes à ses propres rangs. En effet, une petite formation apparentée au PiS, Pologne solidaire (Solidarna Polska), est dirigé par Zbigniew Ziobro, qui s'est signalé par ses ambiguités en tant ministre de la Justice dans des affaires concernant des militants d'extrême droite. À plusieurs reprises il a interféré ou est intervenu publiquement pour s'étonner de la sévérité de la justice. En 2016, il a critiqué la condamnation à dix mois de prison ferme un militant ONR qui avait en 2015 incendié un mannequin à l'effigie d'un juif lors

d'une marche nationaliste à Wrocław, pour souligner que le parquet avait requis une condamnation à des travaux d'intérêt général ${ }^{66}$. La même année, il avait ordonné au parquet de mettre fin aux poursuites engagées pour incitation à la haine raciale envers les Juifs et le Ukrainiens contre un autre militant ONR, ancien prêtre devenu chroniqueur et influenceur sur les réseaux sociaux ${ }^{67}$. Ces ambiguités se sont maintenus par la suite, par exemple

\footnotetext{
${ }^{61}$ « Protestowali przeciwko rasistowskim hasłom na marszu niepodległości. Policja oskarżyła ich o faszyzm », site naTemat.pl, http://natemat.pl/222715,protestowali-przeciwko-rasistowskim-haslom-na-marszu-niepodleglosci-policjaoskarzyla-ich-o-faszyzm consulté le 3 janvier 2018.

${ }^{62}$ http://www.prezydent.pl/aktualnosci/wypowiedzi-prezydenta-rp/inne/art,282,list-prezydenta-do-uczestnikow-marszuniepodleglosci-.html consulté le 2 février 2018.

${ }^{63}$ Ibid.

${ }^{64}$ http://www.fronda.pl/a/robert-bakiewicz-dla-frondy-maszerowali-patrioci-prowokatorzy-osmieszali-marsz-i-darligeby,102484.html consulté le 2 février 2018.

${ }^{65}$ La lettre lue en 2016 est disponible sur le site de la présidence de la République polonaise :

http://www.prezydent.pl/aktualnosci/wypowiedzi-prezydenta-rp/inne/art,282,list-prezydenta-do-uczestnikow-marszuniepodleglosci-.html

66 http://wroclaw.wyborcza.pl/wroclaw/7,35771,21560940,jak-prokurator-bronil-antysemity-ktory-spalil-kukle-zydapoznalismy.html consulté le 2 février 2018.

${ }^{67}$ http://wroclaw.wyborcza.pl/wroclaw/7,35771,22359046,rasista-pod-ochrona-ludzi-ziobry-tylko-u-nas.html consulté le 2 février 2018.
} 
lors d'un scandale qui a éclaté en 2018 après la diffusion sur la chaîne d'opposition TVN 24 d'un reportage sur les milieux néo-nazis qui montrait un petit ressemblement de quelques personnes faisant le salut nazi, arborant des croix gammées et se partageant une pâtisserie décorée d'un cercle blanc ornée d'une croix gammée de gaufrettes en chocolat, sur un fond de nappage rouge. Au grand dam des milieux libéraux, le parquet avait alors, durant plusieurs mois, ouvert une instruction contre l'auteur du reportage pour avoir diffusé des symboles fascistes.

\section{Conclusion : l'échec du managed nationalism à la polonaise ?}

La Marche de l'Indépendance de 2018 s'est tenue dans des circonstances inédites : elle prenait en effet place dans le contexte de la commémoration du centenaire de l'indépendance par l'État polonais, qui a engagé de puissants moyens dans des opérations très nombreuses, qui ne se sont pas limitées à la sphère commémorative stricto sensu. Les transactions entre les organisateurs de la Marche, qui souhaitaient déjà les années précédentes obtenir la présidence d'invités officiels, à commencer par le président Duda, ne s'en sont que complexifiées. À l'approche de la date du 11 novembre, le PiS a fait une proposition qui pourrait résumer son rapport à cet événement, en conviant tous les partis d'opposition à marcher le 11 novembre. Les principaux partis concernés, à commencer par les libéraux de la PO, ont refusé et la Marche s'est décomposé en deux cortèges, sur le même parcours, celui des officiels puis celui des groupes nationalistes. Si le PiS n'est pas parvenu à mettre réellement en difficulté l'opposition, il a en revanche réussi à préempter la marche de l'Indépendance et à reléguer au second plan l'ONR et la Jeunesse panpolonaise. Ainsi, davantage qu'à un arc nationaliste réunissant de manière solidaire droite conservatrice et extrême droite, assiste-t-on davantage à des tentatives de contrôle ou de conditionnement de la sphère nationaliste pour conserver la maitrise de la concurrence politique à droite de la part du PiS. La capacité de ces groupes militants à se réformer pour adopter un nouveau style politique, redéfinir leur image publique et s'engager ouvertement dans la compétition politique avec la création du RN atteste cependant de leurs tentatives de conserver leur autonomie face aux acteurs dominants de la politique polonaise et à faire face, à l'avenir, aux coûts de la participation à la compétition électorale. 